\title{
Oral Administration of Flavonifractor plautii, a Bacteria Increased With Green Tea Consumption, Promotes Recovery From Acute Colitis in Mice via Suppression of IL-17
}

\author{
Ayane Mikami ${ }^{\dagger}$, Tasuku Ogita ${ }^{\dagger}$, Fu Namai, Suguru Shigemori, Takashi Sato and \\ Takeshi Shimosato* \\ Department of Biomolecular Innovation, Institute for Biomedical Sciences, Shinshu University, Nagano, Japan
}

OPEN ACCESS

Edited by:

Clara G. De Los Reyes-Gavilan,

Consejo Superior de Investigaciones

Cientificas (CSIC), Spain

Reviewed by:

Ravinder K. Nagpal,

Florida State University, United States

David Rios-Covian

University of California, San Diego,

United States

*Correspondence:

Takeshi Shimosato

shimot@shinshu-u.ac.jp

tThese authors have contributed equally to this work

Specialty section:

This article was submitted to

Nutrition and Microbes,

a section of the journal

Frontiers in Nutrition

Received: 28 September 2020 Accepted: 24 December 2020

Published: 04 February 2021

Citation:

Mikami A, Ogita T, Namai F,

Shigemori S, Sato $T$ and Shimosato $T$

(2021) Oral Administration of

Flavonifractor plautii, a Bacteria

Increased With Green Tea

Consumption, Promotes Recovery

From Acute Colitis in Mice via

Suppression of IL-17.

Front. Nutr. 7:610946.

doi: 10.3389/fnut.2020.610946
Flavonifractor plautii (FP) has been reported to participate in the metabolism of catechins in the human gut. However, there is limited information on the immune regulatory effects of this bacterium. We confirmed that the administration of green tea increases the abundance of FP in the gut microbiota and investigated the effect of FP in a mouse colitis model. Mice were orally administered FP for 10 consecutive days; colonic inflammation was evaluated daily on the basis of stool consistency, gross rectal bleeding, and body weight. In the dextran sodium sulfate model, FP-exposed animals exhibited lower levels of inflammation and strong inhibition of interleukin (IL)-17 signaling. Moreover, lipoteichoic acid from FP was identified as the active component mediating IL-17 suppression. Thus, oral administration of FP appears to modulate gut inflammation and represents a viable and inexpensive oral microbial therapeutic.

Keywords: Flavonifractor plautii, green tea, inflammatory bowel disease, IL-17, lipoteichoic acid

\section{INTRODUCTION}

Inflammatory bowel disease (IBD) is a generic term for chronic inflammatory disease in the gastrointestinal tract, typified by Crohn's disease and ulcerative colitis (1). IBD patients exhibit macrophage infiltration and excessive activation of tumor necrosis factor alpha (TNF- $\alpha$ ) and interleukin (IL)-17 in local inflammation (2). This results in tissue injury, such as intestinal barrier disruption and ulceration (3), and the development of further hyperimmunity (4). Several studies reported that dysbiosis occurs in IBD patients, involving a reduction in Firmicutes, Bacteroidetes, and commensal bacterial diversity and higher populations of Escherichia coli and Clostridium difficile (5-8). In the last 30 years, the number of IBD patients has been rapidly increasing, mainly in Europe and the United States, and a similar trend in Japan has been observed $(9,10)$. A genetic predisposition and the involvement of environmental factors have been reported in the development of $\operatorname{IBD}(11,12)$. However, in spite of the relatively homogeneous genetic background of the Japanese population, environmental factors seem to affect disease development more strongly than genetic predisposition, as evidenced by the rapid rise in the number of patients. In the 1970s, when the number of patients began to increase rapidly in Japan, Western food featuring high-fat and low-fiber content was pervasive (13), and consequent differences in diet and gut microbiota may have contributed to the occurrence of IBD. Moreover, the use of germ-free mice 
in investigating IBD suggests that alterations in the gut microbiota may be involved in IBD development (14). Intestinal fecal microbiota transplantation has been reported to successfully treat IBD patients (15). These reports suggest that alteration in the gut microbiota may be involved in the onset of $\operatorname{IBD}(16,17)$.

In recent years, it has been reported that dysbiosis is involved not only in IBDs but also in other inflammatory diseases such as obesity and articular rheumatism $(14,18)$. In contrast, a healthy microbiota contributes to the host's health maintenance via the immune system (19). Interestingly, it has become evident that functional enterobacteria play a role in controlling the differentiation and maturation of immune cells in the host intestinal immune system (20). Segmented filamentous bacteria contribute to postnatal maturation of the intestinal immune system; specifically, T-helper-type 17 cell differentiation, immunoglobulin A (IgA) production, and intestinal barrier protection (20). These functions help protect against invading pathogens. For example, we reported that the common enterobacterium Flavonifractor plautii (FP) regulates inflammation in an obesity model (21). FP is a Gram-positive bacteria belonging to the Clostridiales order and is involved in catechin metabolism in the intestines. However, its functionality is poorly understood. In this study, we investigated the effect of the consumption of green tea, a common beverage in Japan, on the abundance of FP in the gut of mice. Following confirmation that the administration of green tea increases the abundance of FP in the gut microbiota, the physiological effects of FP were investigated in an IBD mouse model. Therefore, we investigated the in vitro and in vivo anti-inflammatory effect of FP, as well as the suppression of Th17 immune responses by FP, especially during the recovery phase of an IBD model.

\section{MATERIALS AND METHODS}

\section{Reagents}

Cell culture reagents and supplies were purchased from Thermo Fisher Scientific, Inc. (Waltham, MA, USA, RRID:SCR_008452). Cells were cultured in complete Roswell Park Memorial Institute (RPMI) 1640 medium supplemented with 10\% fetal bovine serum, $1 \%$ non-essential amino acids, and antibiotics $(100 \mathrm{U} / \mathrm{ml}$ penicillin, $100 \mathrm{mg} / \mathrm{ml}$ streptomycin), $25 \mathrm{mM}$ HEPES, $1.0 \mathrm{mM}$ sodium pyruvate, and $0.0035 \% 2$-mercaptoethanol. The green tea used in this study was commercially available in powdered form. All other chemicals were purchased from Nacalai Tesque (Kyoto, Japan, RRID:SCR_013519).

\section{Preparation of FP and Lipoteichoic Acid From FP}

FP was purchased from the American Type Culture Collection (Flavonifractor plautii ATCC ${ }^{\circledR} 29863^{\mathrm{TM}}$, Manassas, VA, USA) and cultured as previously described (22). Briefly, FP was cultured in Gifu Anaerobic Medium (GAM) broth (Nissui Pharmaceutical Co., Ltd., Tokyo, Japan), and the cells were pelleted by centrifugation at $8,000 \times g, 4^{\circ} \mathrm{C}$ for $5 \mathrm{~min}$, and then washed with and resuspended in sterile water to yield a suspension at a density of $1 \times 10^{11}$ colony-forming units $(\mathrm{cfu}) / \mathrm{mL}$. The resulting suspension was lyophilized, and the bacterial cells were stored at $-80^{\circ} \mathrm{C}$ until used for the experiments. Lipoteichoic acid (LTA) was isolated as previously reported (23). For the morphological investigation of FP using scanning electron microscopy (SEM), FP was washed with phosphate-buffered saline (PBS), suspended in PBS, and then placed on a filter (Nanopercolator, JEOL, Tokyo, Japan). The liquid was suctioned using a $10 \mathrm{ml}$ syringe, and the sample was fixed by immersion in $2.5 \%$ glutaraldehyde (TAAB Laboratories Equipment, Ltd., Berks, UK) for $1 \mathrm{~h}$. After fixation, plasma membranes were fixed by lightly washing three times with $0.1 \mathrm{M}$ cacodylate buffer and soaking for $1 \mathrm{~h}$ in $1 \%$ osmium. Following dehydration with ethanol, the samples were soaked in t-butyl alcohol, incubated twice at $52^{\circ} \mathrm{C}$ for $30 \mathrm{~min}$, and stored at $-80^{\circ} \mathrm{C}$. Osmium coatings were carried out using the osmium coater Neoc-AN (Meiwafosis Co., Ltd., Tokyo, Japan). SEM images were obtained using a Miniscope TM4000PlusII (HITACHI High Technologies Corp., Tokyo, Japan) with an accelerating voltage of $15 \mathrm{kV}$.

\section{Mice}

All animal experiments were conducted in accordance with the guidelines for animal experiments of Shinshu University. C57BL/6 mice (7 weeks, female) were obtained from Japan SLC (Hamamatsu, Japan) and housed under controlled conditions of temperature and light. A rodent diet (MF; Oriental Yeast Co., Ltd., Tokyo, Japan) and sterile water were provided ad libitum.

\section{Green Tea Intake in Mice}

C57BL/ 6 mice ( 9 weeks, female) were acclimatized for 2 weeks before the start of the experiments. Mice were divided into two experimental groups ( $n=7-8$ each). There was no significant difference in body weight between the groups. Mice were supplied green tea or sterile water ad libitum for 10 days $(n=7-$ 8 ). The green tea was prepared by dissolving powdered green tea at a concentration of $1 \%$ in boiling sterile water and incubating at $100^{\circ} \mathrm{C}$ for $5 \mathrm{~min}$. The drinking bottles were changed daily. Water intake was measured every day. Body weight and food intake were measured every second day.

\section{Measurement of IL-17 in Feces}

Fecal samples were prepared as previously reported (24). Feces were collected and stored $-80^{\circ} \mathrm{C}$ until analysis. In brief, fecal samples were divided into $20 \mathrm{mg}$ portions and homogenized in $100 \mu \mathrm{L}$ of Halt Protease \& Phosphatase Inhibitor Cocktail $100 \times$ and EDTA $100 \times$ (Thermo Fisher Scientific, Waltham, MA, USA) diluted 1:100. The mixture was centrifuged at 400 $\times g$ for $5 \mathrm{~min}$ at $4^{\circ} \mathrm{C}$. The supernatant fluid was collected, and IL-17 levels were measured by ELISA (Mouse DuoSet ELISA kit, R\&D Systems, Minneapolis, MN, USA) according to the manufacturer's instructions.

\section{Abundance of FP in Feces}

DNA from fecal samples was extracted and purified using a QIAamp Fast DNA Stool Mini kit (Qiagen, Valencia, CA, USA) according to the manufacturer's protocol. FP abundance was measured by quantitative PCR (qPCR) as described previously $(22,25)$. Quantification of the target FP 16 S ribosomal DNA 
(rDNA) levels was performed using the $\Delta \Delta \mathrm{Ct}$ method, with normalization to the total $16 \mathrm{~S}$ rDNA content. External pure FP culture or feces-based FP standards were included in each run to construct a standard curve. The concentration ( $\mathrm{cfu} / \mathrm{mg}$ ) of FP in each sample was calculated by comparing the Ct of the sample with that of the standard curve. Primers and cycling conditions were as described previously (22).

\section{DSS-Acute Colitis Model}

C57BL/6 mice ( 9 weeks, female) were acclimatized for 2 weeks before the start of experiments. After acclimation, mice were divided into two experimental groups ( $n=6$ each, housed at 6 mice/cage). There was no significant difference in body weight between the groups. Day 0 of the untreated group $(n=6)$ was used as a baseline. Mice were exposed to $3 \%(\mathrm{w} / \mathrm{w})$ dextran sodium sulfate (DSS) (M.W. 36,000-50,000 Da; MP Biomedicals, Aurora, OH, USA) in the drinking water ad libitum for 5 days. From day 5, they were given sterile water without DSS $(26,27)$. FP $\left(10^{8} \mathrm{cfu} /\right.$ mouse $)$ or PBS was administered via intragastric (i.g.) administration from day 0 to $9(n=12-19)$. The experimental schedule is shown in Figure 2C. During the experiment, body weight and Disease Activity Index (DAI) scores of the severity of colitis symptoms were measured daily (28). Body weight was expressed as a percentage of the initial (day 0) body weight. The DAI score is a combined score of body weight differences, diarrhea, and bloody stools and was evaluated according to a previously reported method (28). On days $0,6,8$, and 10 , mice were sacrificed, and measurements of colon length were obtained. Colon length was measured from the ileocecal valve to the end of the lower rectum. IL-17, IL-23, and IL-10 concentrations in colon homogenates were evaluated by ELISA (Mouse DuoSet ELISA kit, R\&D Systems) according to the manufacturer's instructions. The cytokine levels per colon tissue are presented as $\mathrm{pg} / \mathrm{mg}$ tissue protein in each sample.

\section{IL-17 Regulation Assay}

Splenocytes $\left(1.0 \times 10^{7}\right.$ cells $\left./ \mathrm{mL}\right)$ from C57BL/ 6 mice (6 weeks, female) were stimulated by $50 \mathrm{ng} / \mathrm{mL}$ interleukin 6 (IL) -6 (R\&D Systems) and $2.5 \mathrm{ng} / \mathrm{mL}$ transforming growth factor (TGF)- $\beta 1$ (R\&D Systems). At the same time, medium FP $\left(10^{8} \mathrm{cfu} / \mathrm{mL}\right)$, heat-killed FP $\left(10^{8} \mathrm{cfu} / \mathrm{mL}\right)$, FP-LTA (10 $\mu \mathrm{g} /$ well), and/or LTA from Staphylococcus aureus (SA-LTA; Sigma Aldrich, St. Louis, $\mathrm{MO}$, USA) were added. Cultures were carried out for $72 \mathrm{~h}$ at $37^{\circ} \mathrm{C}$ in an atmosphere of $5 \% \mathrm{CO}_{2}$. The culture supernatants were measured using the above-described ELISA kits.

\section{Statistical Analysis}

All statistical analyses were performed using GraphPad Prism7 (GraphPad Software, Inc., La Jolla, CA, USA). In vitro data are presented as the mean \pm standard deviation (SD). In vivo data are presented as the mean \pm standard error of mean (SE). The statistical significance $(p<0.05)$ of differences was determined by a Student's $t$-test or a one-way analysis of variance (ANOVA).

\section{RESULTS}

\section{Effects of Green Tea Consumption on Fecal FP Abundance and IL-17 Production}

The mice were supplied distilled water or green tea every day for 10 days (Figure 1A). There were no differences in body weight, water intake, and food intake between groups (Figures 1B-D). On days 8 and 10, feces were collected, and FP abundance (Figures 1E,F) were measured. The relative abundance of FP in feces was higher in the green tea supplementation group on day 8 (Figure 1F). The fecal IL-17 levels were not significantly altered by green tea supplementation (data not shown).

\section{Morphological Observation of FP}

FP has been reported as a motile, Gram-positive, non-sporeforming bacillus (29). However, there have been no reports describing the morphology of FP. In this study, we observed FP using SEM; FP appeared as a bacillus that ranged in length from 1 to $8 \mu \mathrm{m}$. The surface of the cell wall was rough and lacked flagella (Figures 2A,B).

\section{FP Alleviates DSS-Induced Acute Colitis Symptoms}

To evaluate the effects on FP on colitis symptoms, we conducted in vivo experiments using a DSS-induced acute colitis model (Figure 2C). Colons were collected on days $0,6,8$, and 10 to observe temporal differences in internal colitis symptoms. The severity of colitis was evaluated using body weight (Figure 2D), DAI score (Figure 2E), and colon length (Figure 2F). Significant differences in body weight between the PBS and FP groups were observed on days 8-10 (Figure 2D). FP treatment significantly lowered DAI scores on days 8-10 (Figure 2E). Diarrhea and bloody stool scores were improved in mice administered FP on days 3 and 5, respectively (data not shown). Colon length of the FP group was longer compared to PBS on day 8 (Figure 2F). Significant improvement in the rate of body weight difference was observed in the FP group on days 8-10. In the FP group, a significant reversal of DSS-induced colon shortening was observed on day 8 .

\section{FP Suppressed IL-17 Production in the Colon}

Th17 cells massively infiltrate the intestine of IBD patients, where they mainly produce IL-17A. IL-17 signaling is triggered and amplified during the inflammatory process (30). IL-23 is a cytokine that maintains and proliferates Th17 cells in IBD (31). Therefore, we measured IL-17, IL-23, and IL-10 levels in colon tissues (Figures 3A-C). On day 10, IL-17 level was significantly lower in the colon of the FP group $(p=0.006)$ (Figure 3A). However, IL-23 and IL-10 production in colon tissues did not differ between groups (Figures 3B,C). Thus, the FP group exhibited lower IL-17 levels that were unrelated to IL-10 signaling. IL-23 is a Th17-related cytokine that activates gut inflammation, and its production is elevated in IBD patients (32). IL-10 is also known as a cytokine related to IL-17. These cytokines often show opposite expression patterns in IBD models; specifically, IL-17 levels are elevated in colitis mice (33). 


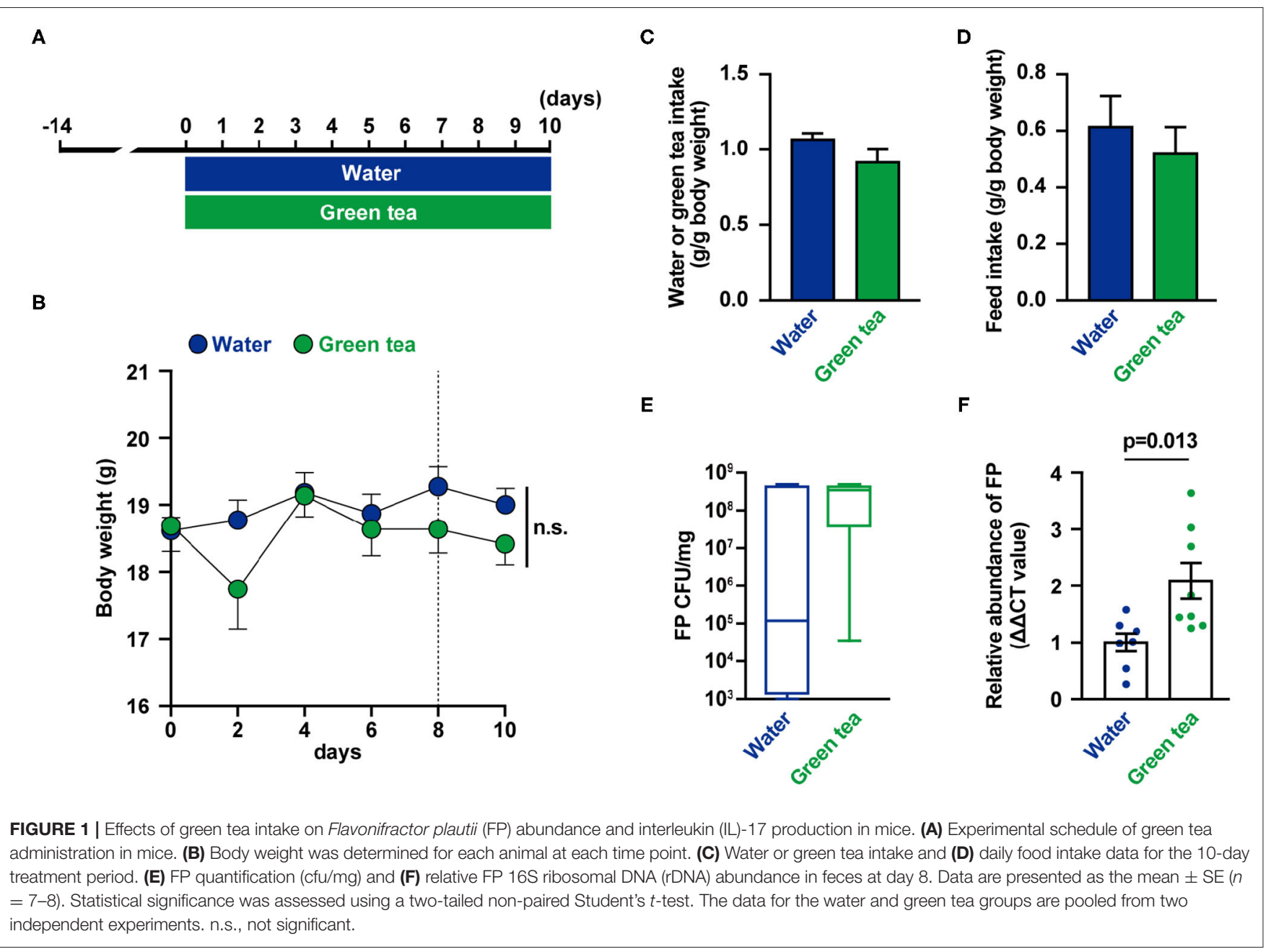

Consistent with this, there were no significant differences in IL-23 and IL-10 production associated with FP treatment on either day.

\section{FP-LTA Suppressed IL-17 Production}

Son et al. (34) reported that bacterial LTA can reduce proliferation of effector $\mathrm{CD} 4{ }^{+} \mathrm{T}$ cells. Therefore, we investigated the effect of FP-LTA on IL-17 suppression in vitro. Specifically, we focused on LTA, which is a major cell wall component of Grampositive bacteria such as FP. Treatment of mouse splenocytes with the FP or SA-LTA, as well as live-FP and HK-FP, resulted in the inhibition of IL-17 production (Figure 4).

\section{DISCUSSION}

Although FP has been reported to be involved in the metabolism of catechins $(35,36)$, the impact of catechins on the relative abundance of FP has not been clarified. Here, we investigated the relationship between FP and catechins in mice administered green tea, a catechin-rich material. Catechins are antioxidants found in a variety of foods and constitute up to $42 \%$ of green tea by dry weight. The major catechins in green tea are (-)-epigallocatechin-3-gallate, (-)-epicatechin-3-gallate, (-)epigallocatechin, and (-)-epicatechin (37). These catechins have been reported to exert a variety of beneficial effects including antioxidant (38), anti-inflammatory (39), antiobesity (40), and anticancer effects $(37,41)$. Green tea has also been reported to suppress IL-17 expression in rheumatoid arthritis, periodontitis, and IBD model mice (42). Interestingly, we showed that the abundance of FP was higher on day 8 with green tea drinking.

Next, we aimed to investigate the use of FP as part of the host's commensal gut bacteria in mitigating acute colitis. A DSSinduced colitis model was used to reproduce IBD symptoms and validate the efficacy of FP strains in reducing intestinal inflammation. Shortening of colon length is a symptom of IBD (43) and was improved on day 8 in mice fed FP. This suggests that FP relieves DSS-induced colitis symptoms. Recovery of these symptoms was not observed until days 6 and 7. Body weight and DAI scores did not differ between groups on day 6. Therefore, FP may promote recovery rather than suppress worsening of colitis symptoms. To investigate the responsible mechanisms, we focused on Th17. Th17 cells produce IL-17 and are the main immune cell involved in mucosal immunity and act to defend against extracellular bacterial and fungal infections. 
A

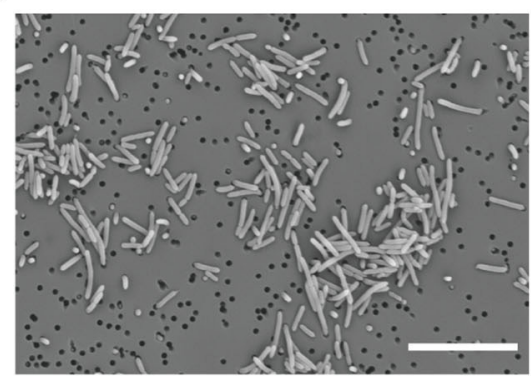

C

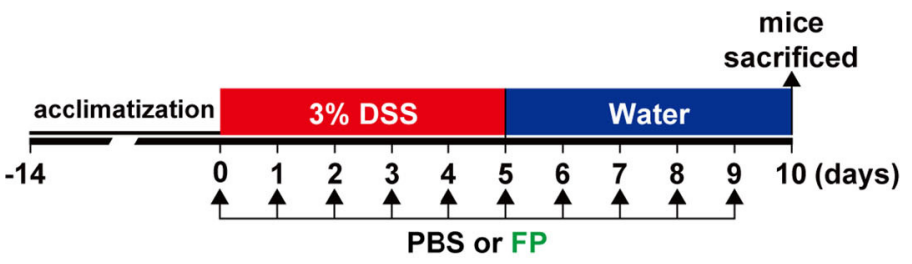

(i.g.)

D

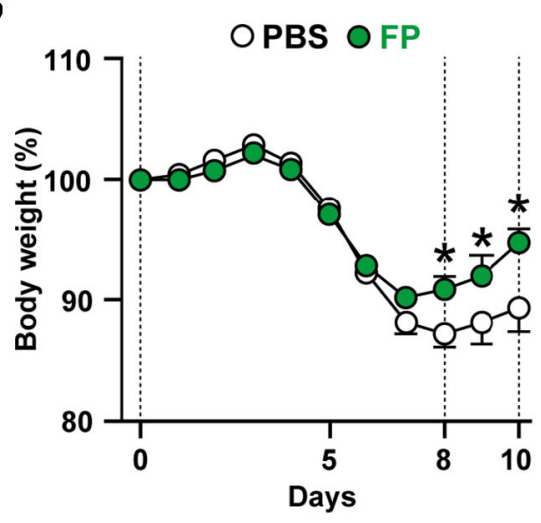

$\mathbf{F}$

n.s.

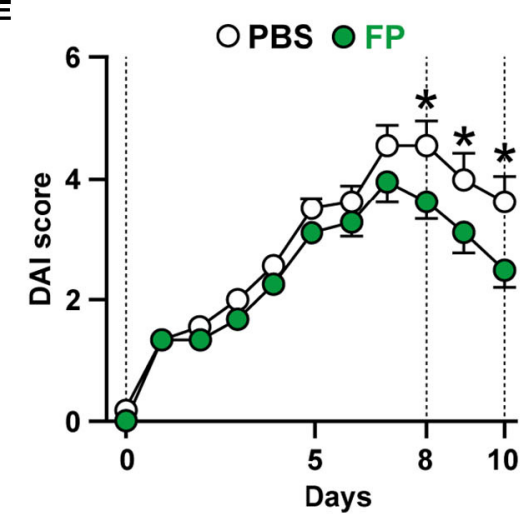

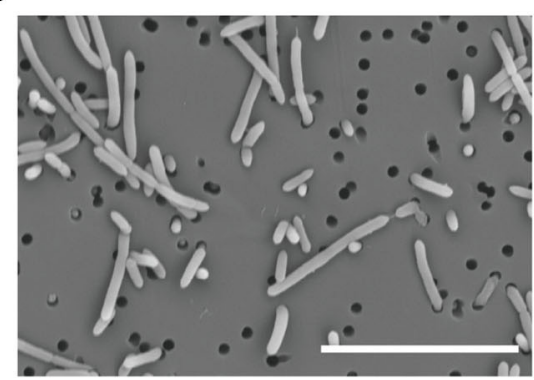

mice

sacrificed

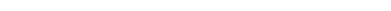

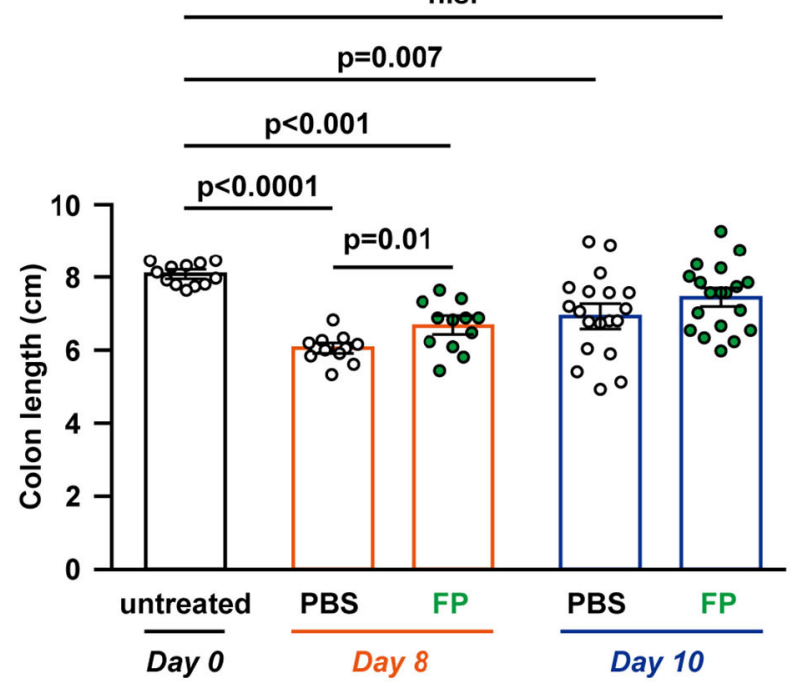

FIGURE 2 | (A,B) Scanning electron microscopy (SEM) image of Flavonifractor plautii (FP). SEM photographs: (A) $\times 2,500$ and (B) $\times 5,000$ of FP. Scale bar $=10 \mu \mathrm{m}$. Effects of oral FP administration on dextran sodium sulfate (DSS)-induced colitis in mice. (C) Experimental schedule. (D) Body weight measurements and colitis symptoms as scored by (E) Disease Activity Index (DAl) or (F) colon length on days 0, 8, and 10. Colon length was measured from the base of the cecum to the anal constriction. $n=12-19$ mice per group. Statistical significance was assessed using a two-tailed non-paired Student's $t$-test. The data for the phosphate-buffered saline (PBS) and FP groups are pooled from three independent experiments. n.s., not significant. 
A

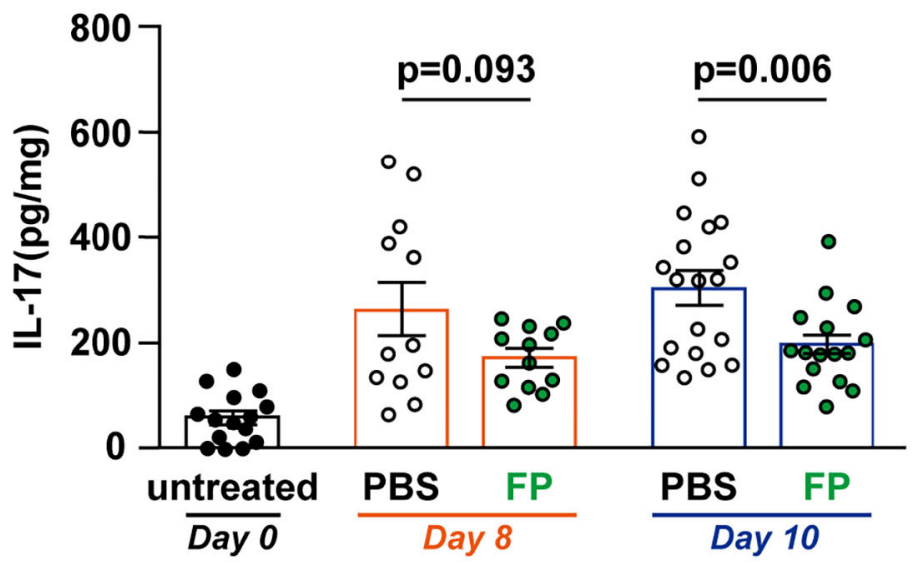

B

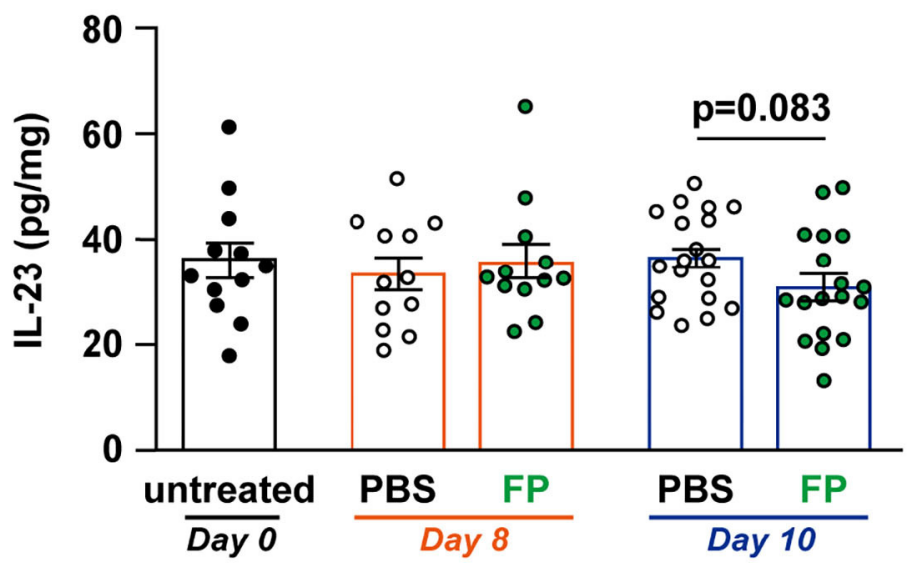

C

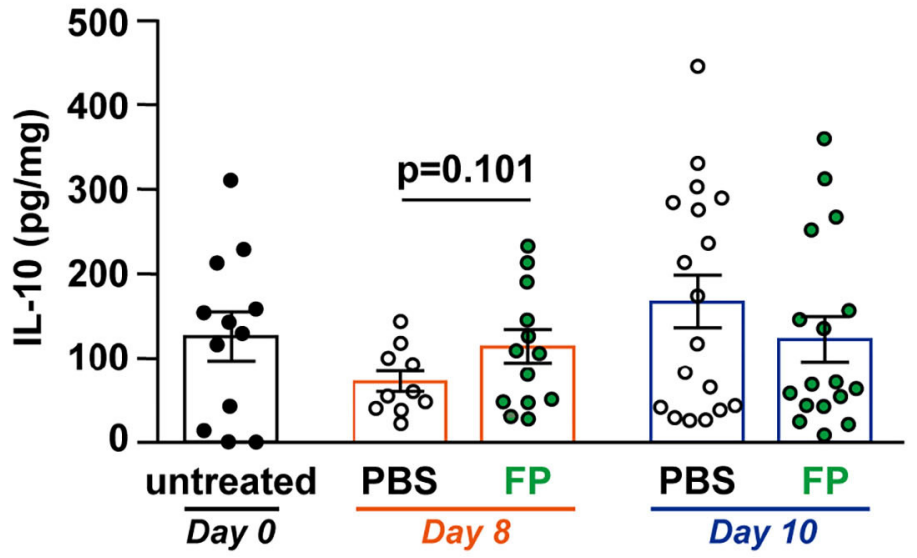

FIGURE 3 | Effect of Flavonifractor plautii (FP) on interleukin (IL)-17, IL-10, and IL-23 levels in the colon tissue. (A) IL-17, (B) IL-23, or (C) IL-10 levels were evaluated in colon tissue homogenates collected on days 0,8 , and 10. Data are presented as the mean \pm SE $(n=12-16)$. Statistical significance was assessed using a two-tailed non-paired Student's $t$-test. The data for the phosphate-buffered saline (PBS) and FP groups are pooled from three independent experiments. 


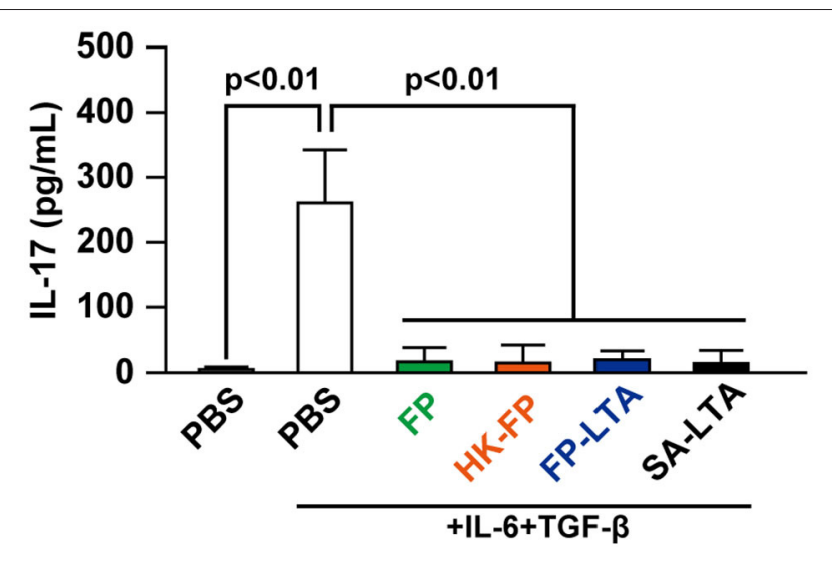

FIGURE 4 | The effect of LTA on interleukin (IL)-17 levels in mouse splenocytes. IL-17 production in mouse splenocytes was determined in the presence and absence of IL-6/transforming growth factor (TGF)- $\beta$ and various sources of LTA. Cellular supernatants were collected, and IL-17 levels were measured with ELISA. Values represent means, and error bars indicate the SD of three independent experiments. $p<0.01$ by one-way analysis of variance followed by Tukey's multiple comparison test. At least three independent experiments were conducted in triplicate. HK, heat killed; SA, Staphylococcus aureus, LTA, lipoteichoic acid.

Overexpression of IL-17 has been reported to be associated with IBD pathogenesis and is abundantly found in inflamed IBD gut mucosa (44-47). We observed that IL-17 production in the colon tissue was lower in the FP group on days 6 and 10, indicating that FP alleviates mucosal damage by suppressing the overexpression of IL-17.

In the in vitro experiment, FP suppressed the production of IL-17. When splenocytes are stimulated with IL-6 and TGF- $\beta$, the main source of IL-17 is Th17 (IL-17 ${ }^{+}$and CD4 ${ }^{+}$T cells) (48). This result suggests that FP suppressed IL-17 production from Th17 cells. In addition, LTA preparations derived from FP (FPLTA) also induced a decline in IL-17 production. LTA is a cell wall component of Gram-positive bacteria, and administration of LTA-deficient Lactobacillus acidophilus to mice decreased inflammatory signals (49). In contrast, LTA also exhibits antiinflammatory effects by suppressing neutrophil migration (50). Since previous studies have not reported that LTA suppresses IL-17 expression, this result may be specific to bacterial-LTA.

FP is reported to be a flavonoid-degrading bacteria that is present in all mammals and has the ability to metabolize quercetin and produce butyrate (29). Interestingly, Kasai et al. reported that FP was detected more frequently in stool samples from normal subjects $(0.22 \%)$ than obese subjects $(0.06 \%)$ in the Japanese population (51). It is well-known that inflammation in obesity is associated with microbiota composition (52). Therefore, it is possible that FP contributes to health maintenance. In this study, IBD symptoms were suppressed by FP. In contrast, some reports have suggested the involvement of FP in intestinal disease (53-55). Dinitrobenzene sulfonic acid (DNBS)-induced ulcerative colitis mice showed a reduction in the relative abundance of FP (56). These reports suggest that among IBD, FP may promote recovery from ulcerative colitis. While FP is not thought to exhibit toxicity, further investigation of its safety is necessary (57).

In a DSS-induced acute colitis model, improvements in the disease state may involve reduced gut inflammation (58). An improvement in the percentage of body weight difference in the FP-treated group was observed on days 8, 9, and 10. No differences in food or water intake were observed in both groups. Therefore, accelerated weight regain was considered to be a consequence of the effect of FP on the host's immune system. Lenzen et al. reported that the restoration of DSSinduced colitis involves suppression of inflammatory cytokine expression, epithelial apoptosis of the colon, intestinal barrier rupture, and promotion of mucosal restoration and various transport mechanisms (59). FP may have contributed to these functions, and the link between green tea consumption and improved gut health is increasingly coming to the fore. This botanical beverage may hold benefits for people at high risk of inflammatory diseases.

\section{DATA AVAILABILITY STATEMENT}

The raw data supporting the conclusions of this article will be made available by the authors, without undue reservation.

\section{ETHICS STATEMENT}

The animal study was reviewed and approved by the Committee for Animal Experiments of Shinshu University. Written informed consent was obtained from the owners for the participation of their animals in this study.

\section{AUTHOR CONTRIBUTIONS}

TO and FN conceived of and designed the experiments. AM and TO conducted the experiments. SS and TSa contributed reagents, materials, and analytical tools. AM and TSh wrote the paper. TSh supervised the work. All authors reviewed the manuscript.

\section{FUNDING}

This study was funded by a Grant-in-Aid for Young Scientists (B) (No. 17K15268) and the Fund of Nagano Prefecture to Promote Scientific Activity.

\section{ACKNOWLEDGMENTS}

We thank Chikako Miyazaki of the Institute for Biomedical Sciences, Shinshu University for the technical support. We also thank the Research Center for Supports to Advanced Science, Shinshu University for use of their facilities. 


\section{REFERENCES}

1. Mijac D, Vukovic-Petrovic I, Mijac V, Perovic V, Milic N, Djuranovic S, et al. MDR1 gene polymorphisms are associated with ulcerative colitis in a cohort of Serbian patients with inflammatory bowel disease. PLOS ONE. (2018) 13:e0194536. doi: 10.1371/journal.pone.0194536

2. Lee SY, Lee SH, Yang EJ, Kim EK, Kim JK, Shin DY, et al. Metformin ameliorates inflammatory bowel disease by suppression of the STAT3 signaling pathway and regulation of the between Th17/treg balance. PLoS ONE. (2015) 10:e0135858. doi: 10.1371/journal.pone.0135858

3. Mikhailov TA, Furner SE. Breastfeeding and genetic factors in the etiology of inflammatory bowel disease in children. World J Gastroenterol. (2009) 15:270-9. doi: 10.3748/wjg.15.270

4. Kantso B, Simonsen J, Hoffmann S, Valentiner-Branth P, Petersen AM, Jess T. Inflammatory bowel disease patients are at increased risk of invasive pneumococcal disease: A Nationwide Danish Cohort Study 1977-2013. Am J Gastroenterol. (2015) 110:1582-7. doi: 10.1038/ajg.2015.284

5. Barnich N, Carvalho FA, Glasser AL, Darcha C, Jantscheff P, Allez M, et al. CEACAM6 acts as a receptor for adherent-invasive E. coli, supporting ileal mucosa colonization in Crohn disease. J Clin Invest. (2007) 117:1566-74. doi: $10.1172 /$ jci30504

6. Ott SJ, Musfeldt M, Wenderoth DF, Hampe J, Brant O, Folsch UR, et al. Reduction in diversity of the colonic mucosa associated bacterial microflora in patients with active inflammatory bowel disease. Gut. (2004) 53:685-93. doi: 10.1136/gut.2003.025403

7. Rodemann JF, Dubberke ER, Reske KA, Seo DH, Stone CD. Incidence of Clostridium difficile infection in inflammatory bowel disease. Clin Gastroenterol Hepatol. (2007) 5:339-44. doi: 10.1016/j.cgh.2006.12.027

8. Zeng MY, Inohara N, Nunez G. Mechanisms of inflammation-driven bacterial dysbiosis in the gut. Mucosal Immunol. (2017) 10:18-26. doi: $10.1038 / \mathrm{mi} .2016 .75$

9. Kitahora T, Utsunomiya T, Yokota A. Epidemiological study of ulcerative colitis in Japan: incidence and familial occurrence. The Epidemiology Group of the Research Committee of Inflammatory Bowel Disease in Japan. $J$ Gastroenterol. (1995) 30(Suppl. 8):5-8.

10. Shoda R, Matsueda K, Yamato S, Umeda N. Epidemiologic analysis of Crohn disease in Japan: increased dietary intake of $n-6$ polyunsaturated fatty acids and animal protein relates to the increased incidence of Crohn disease in Japan. Am J Clin Nutr. (1996) 63:741-5. doi: 10.1093/ajcn/63.5.741

11. Yamamoto-Furusho JK. Genetic factors associated with the development of inflammatory bowel disease. World J Gastroenterol. (2007) 13:5594-7. doi: 10.3748/wjg.v13.i42.5594

12. Yamamoto-Furusho JK, Fonseca-Camarillo G. Genetic markers associated with clinical outcomes in patients with inflammatory bowel disease. Inflamm Bowel Dis. (2015) 21:2683-95. doi: 10.1097/mib.0000000000000500

13. Asakura H, Suzuki K, Kitahora T, Morizane T. Is there a link between food and intestinal microbes and the occurrence of Crohn's disease and ulcerative colitis? J Gastroenterol Hepatol. (2008) 23:1794-801. doi: 10.1111/j.1440-1746.2008.05681.x

14. Kuhn R, Lohler J, Rennick D, Rajewsky K, Muller W. Interleukin10-deficient mice develop chronic enterocolitis. Cell. (1993) 75:263-74. doi: 10.1016/0092-8674(93)80068-p

15. Thomas H. IBD: FMT induces clinical remission in ulcerative colitis. Nat Rev Gastroenterol Hepatol. (2017) 14:196. doi: 10.1038/nrgastro.2017.27

16. Balish E, Warner T. Enterococcus faecalis induces inflammatory bowel disease in interleukin-10 knockout mice. Am J Pathol. (2002) 160:2253-7. doi: 10.1016/s0002-9440(10)61172-8

17. Taurog JD, Richardson JA, Croft JT, Simmons WA, Zhou M, FernandezSueiro JL, et al. The germfree state prevents development of gut and joint inflammatory disease in HLA-B27 transgenic rats. J Exp Med. (1994) 180:2359-64. doi: 10.1084/jem.180.6.2359

18. Beyer K, Zaura E, Brandt BW, Buijs MJ, Brun JG, Crielaard W, et al. Subgingival microbiome of rheumatoid arthritis patients in relation to their disease status and periodontal health. PLoS ONE. (2018) 13:e0202278. doi: 10.1371/journal.pone.0202278

19. Amenyogbe N, Kollmann TR, Ben-Othman R. Early-life host-microbiome interphase: the key frontier for immune development. Front Pediatr. (2017) 5:111. doi: $10.3389 /$ fped.2017.00111
20. Flannigan KL, Denning TL. Segmented filamentous bacteria-induced immune responses: a balancing act between host protection and autoimmunity. Immunology. (2018) 154:537-46 doi: 10.1111/imm.12950

21. Mikami A, Ogita T, Namai F, Shigemori S, Sato T, Shimosato T. Oral administration of Flavonifractor plautii attenuates inflammatory responses in obese adipose tissue. Mol Biol Rep. (2020) 47:6717-25. doi: 10.1007/s11033-020-05727-6

22. Ogita T, Yamamoto Y, Mikami A, Shigemori S, Sato T, Shimosato T. Oral Administration of Flavonifractor plautii strongly suppresses Th2 immune responses in mice. Front Immunol. (2020) 11:379. doi: 10.3389/fimmu.2020.00379

23. Mistretta N, Brossaud M, Telles F, Sanchez V, Talaga P, Rokbi B. Glycosylation of Staphylococcus aureus cell wall teichoic acid is influenced by environmental conditions. Sci Rep. (2019) 9:3212. doi: 10.1038/s41598-019-39929-1

24. Jovanovic M, Simovic Markovic B, Gajovic N, Jurisevic M, Djukic A, Jovanovic I, et al. Metabolic syndrome attenuates ulcerative colitis: correlation with interleukin-10 and galectin-3 expression. World J Gastroenterol. (2019) 25:6465-82. doi: 10.3748/wjg.v25.i43.6465

25. Ahlroos T, Tynkkynen S. Quantitative strain-specific detection of Lactobacillus rhamnosus GG in human faecal samples by real-time PCR. $J$ Appl Microbiol. (2009) 106:506-14. doi: 10.1111/j.1365-2672.2008.04018.x

26. Namai F, Shigemori S, Ogita $T$, Sato $T$, Shimosato T. Microbial therapeutics for acute colitis based on genetically modified Lactococcus lactis hypersecreting IL-1Ra in mice. Exp Mol Med. (2020) 52:1627-36. doi: 10.1038/s12276-020-00507-5

27. Shigemori S, Namai F, Ogita T, Sato T, Shimosato T. Oral priming with oligodeoxynucleotide particles from Lactobacillus rhamnosus GG attenuates symptoms of dextran sodium sulfate-induced acute colitis in mice. Anim Sci J. (2020) 91:e13468. doi: 10.1111/asj.13468

28. Shigemori S, Watanabe T, Kudoh K, Ihara M, Nigar S, Yamamoto Y, et al. Oral delivery of Lactococcus lactis that secretes bioactive heme oxygenase-1 alleviates development of acute colitis in mice. Microb Cell Fact. (2015) 14:189. doi: 10.1186/s12934-015-0378-2

29. Carlier JP, Bedora-Faure M, K'Ouas G, Alauzet C, Mory F. Proposal to unify Clostridium orbiscindens Winter et al. 1991 and Eubacterium plautii (Seguin 1928) Hofstad and Aasjord 1982, with description of Flavonifractor plautii gen. nov., comb. nov., and reassignment of Bacteroides capillosus to Pseudoflavonifractor capillosus gen. nov., comb. nov. Int J Syst Evol Microbiol. (2010) 60(Pt 3):585-90. doi: 10.1099/ijs.0.01 6725-0

30. Galvez J. Role of Th17 cells in the pathogenesis of human IBD. ISRN Inflamm. (2014) 2014:928461. doi: 10.1155/2014/928461

31. Weaver CT, Elson CO, Fouser LA, Kolls JK. The Th17 pathway and inflammatory diseases of the intestines, lungs, and skin. Annu Rev Pathol. (2013) 8:477-512. doi: 10.1146/annurev-pathol-011110-130318

32. Neurath MF. IL-23 in inflammatory bowel diseases and colon cancer. Cytokine Growth Factor Rev. (2019) 45:1-8. doi: 10.1016/j.cytogfr.2018.12.002

33. Taghipour N, Mosaffa N, Aghdaei HA, Rostami-Nejad M, Weinstock JV, Shahnavaz S, et al. Immunomodulatory effect of Syphacia obvelata in treatment of experimental DSS-induced colitis in mouse model. Sci Rep. (2019) 9:19127. doi: 10.1038/s41598-019-55552-6

34. Son YM, Song KD, Park SM, Han SH, Yun CH. Lipoteichoic acid suppresses effector T cells induced by Staphylococcus aureus-pulsed dendritic cells. J Microbiol Biotechnol. (2013) 23:1023-30. doi: 10.4014/jmb.1302. 02009

35. Braune A, Blaut M. Bacterial species involved in the conversion of dietary flavonoids in the human gut. Gut Microbes. (2016) 7:216-34. doi: 10.1080/19490976.2016.1158395

36. Takagaki A, Nanjo F. Bioconversion of (-)-epicatechin, (+)-epicatechin, (-)catechin, and (+)-catechin by (-)-epigallocatechin-metabolizing bacteria. Biol Pharm Bull. (2015) 38:789-94. doi: 10.1248/bpb.b14-00813

37. Singh BN, Shankar S, Srivastava RK. Green tea catechin, epigallocatechin-3gallate (EGCG): mechanisms, perspectives and clinical applications. Biochem Pharmacol. (2011) 82:1807-21. doi: 10.1016/j.bcp.2011.07.093

38. Venkatakrishnan K, Chiu HF, Cheng JC, Chang YH, Lu YY, Han YC, et al. Comparative studies on the hypolipidemic, antioxidant and hepatoprotective activities of catechin-enriched green and oolong tea in a double-blind clinical trial. Food Funct. (2018) 9:1205-13. doi: 10.1039/c7fo01449j 
39. Wu H, Xie Y, Xu Y, Hu Z, Wan X, Huang H, et al. Protective effect of Epicatechin on APAP-induced acute liver injury of mice through antiinflammation and apoptosis inhibition. Nat Prod Res. (2020) 34:855-8. doi: 10.1080/14786419.2018.1503261

40. Yamashita S, Hirashima A, Lin IC, Bae J, Nakahara K, Murata M, et al. Saturated fatty acid attenuates anti-obesity effect of green tea. Sci Rep. (2018) 8:10023. doi: 10.1038/s41598-018-28338-5

41. Shankar S, Ganapathy S, Hingorani SR, Srivastava RK. EGCG inhibits growth, invasion, angiogenesis and metastasis of pancreatic cancer. Front Biosci. (2008) 13:440-52. doi: 10.2741/2691

42. Barbalho SM, Bosso H, Salzedas-Pescinini LM, de Alvares Goulart R. Green tea: a possibility in the therapeutic approach of inflammatory bowel diseases? Green tea and inflammatory bowel diseases. Complement Ther Med. (2019) 43:148-53. doi: 10.1016/j.ctim.2019.01.015

43. Yoda K, Miyazawa K, Hosoda M, Hiramatsu M, Yan F, He F. Lactobacillus GG-fermented milk prevents DSS-induced colitis and regulates intestinal epithelial homeostasis through activation of epidermal growth factor receptor. Eur J Nutr. (2014) 53:105-15. doi: 10.1007/s00394-013-0506-x

44. Bettelli E, Carrier Y, Gao W, Korn T, Strom TB, Oukka M, et al. Reciprocal developmental pathways for the generation of pathogenic effector TH17 and regulatory T cells. Nature. (2006) 441:235-8. doi: 10.1038/nature04753

45. Catana CS, Berindan Neagoe I, Cozma V, Magdas C, Tabaran F, Dumitrascu DL. Contribution of the IL-17/IL-23 axis to the pathogenesis of inflammatory bowel disease. World J Gastroenterol. (2015) 21:5823-30. doi: 10.3748/wjg.v21.i19.5823

46. Hundorfean G, Neurath MF, Mudter J. Functional relevance of T helper 17 (Th17) cells and the IL-17 cytokine family in inflammatory bowel disease. Inflamm Bowel Dis. (2012) 18:180-6. doi: 10.1002/ibd.21677

47. Wang X, Zhao J, Qin L. VEGF-C mediated enhancement of lymphatic drainage reduces intestinal inflammation by regulating IL-9/IL-17 balance and improving gut microbiota in experimental chronic colitis. Am J Transl Res. (2017) 9:4772-84.

48. Ogita T, Nakashima M, Morita H, Saito Y, Suzuki T, Tanabe S. Streptococcus thermophilus ST28 ameliorates colitis in mice partially by suppression of inflammatory Th17 cells. J Biomed Biotechnol. (2011) 2011:378417. doi: $10.1155 / 2011 / 378417$

49. Saber R, Zadeh M, Pakanati KC, Bere P, Klaenhammer T, Mohamadzadeh M. Lipoteichoic acid-deficient Lactobacillus acidophilus regulates downstream signals. Immunotherapy. (2011) 3:337-47. doi: 10.2217/imt.10.119

50. Alves-Filho JC, Freitas A, Souto FO, Spiller F, Paula-Neto H, Silva JS, et al. Regulation of chemokine receptor by Toll-like receptor 2 is critical to neutrophil migration and resistance to polymicrobial sepsis. Proc Natl Acad Sci U S A. (2009) 106:4018-23. doi: 10.1073/pnas.0900196106

51. Kasai C, Sugimoto K, Moritani I, Tanaka J, Oya Y, Inoue H, et al. Comparison of the gut microbiota composition between obese and non-obese individuals in a Japanese population, as analyzed by terminal restriction fragment length polymorphism and next-generation sequencing. BMC Gastroenterol. (2015) 15:100. doi: 10.1186/s12876-015-0330-2

52. Boulange CL, Neves AL, Chilloux J, Nicholson JK, Dumas ME. Impact of the gut microbiota on inflammation, obesity, and metabolic disease. Genome Med. (2016) 8:42. doi: 10.1186/s13073-016-0303-2

53. Armstrong $\mathrm{H}$, Alipour $\mathrm{M}$, Valcheva $\mathrm{R}$, Bording-Jorgensen $\mathrm{M}$, Jovel J, Zaidi D, et al. Host immunoglobulin G selectively identifies pathobionts in pediatric inflammatory bowel diseases. Microbiome. (2019) 7:1. doi: 10.1186/s40168-018-0604-3

54. Gupta A, Dhakan DB, Maji A, Saxena R, P KV, Mahajan S, et al. Association of Flavonifractor plautii, a flavonoid-degrading bacterium, with the gut microbiome of colorectal cancer patients in India. mSystems. (2019) 4:e0043819. doi: 10.1128/mSystems.00438-19

55. Hollister EB, Oezguen N, Chumpitazi BP, Luna RA, Weidler EM, RubioGonzales $\mathrm{M}$, et al. Leveraging human microbiome features to diagnose and stratify children with irritable bowel syndrome. J Mol Diagn. (2019) 21:44961. doi: 10.1016/j.jmoldx.2019.01.006

56. Pagano E, Romano B, Iannotti FA, Parisi OA, D’Armiento M, Pignatiello $\mathrm{S}$, et al. The non-euphoric phytocannabinoid cannabidivarin counteracts intestinal inflammation in mice and cytokine expression in biopsies from UC pediatric patients. Pharmacol Res. (2019) 149:104464. doi: 10.1016/j.phrs.2019.104464

57. Atarashi K, Tanoue T, Oshima K, Suda W, Nagano Y, Nishikawa H, et al. Treg induction by a rationally selected mixture of Clostridia strains from the human microbiota. Nature. (2013) 500:232-6. doi: 10.1038/nature12331

58. Ganji-Arjenaki M, Rafieian-Kopaei M. Probiotics are a good choice in remission of inflammatory bowel diseases: a meta-analysis and systematic review. J Cell Physiol. (2018) 233:2091-103. doi: 10.1002/jcp.25911

59. Lenzen H, Qian J, Manns MP, Seidler U, Jorns A. Restoration of mucosal integrity and epithelial transport function by concomitant anti-TNFalpha treatment in chronic DSS-induced colitis. J Mol Med (Berl). (2018) 96:831-43. doi: 10.1007/s00109-018-1658-1

Conflict of Interest: The authors declare that the research was conducted in the absence of any commercial or financial relationships that could be construed as a potential conflict of interest.

Copyright (C) 2021 Mikami, Ogita, Namai, Shigemori, Sato and Shimosato. This is an open-access article distributed under the terms of the Creative Commons Attribution License (CC BY). The use, distribution or reproduction in other forums is permitted, provided the original author(s) and the copyright owner(s) are credited and that the original publication in this journal is cited, in accordance with accepted academic practice. No use, distribution or reproduction is permitted which does not comply with these terms. 